\title{
Utilisation de la bibliothèque, de la documentation écrite et audiovisuelle au niveau collégial et relations entre le personnel préposé aux ressources et les usagers
}

\author{
Jacques Laliberté
}

Volume 26, numéro 2, juin 1980

URI : https://id.erudit.org/iderudit/1054249ar

DOI : https://doi.org/10.7202/1054249ar

Aller au sommaire du numéro

Éditeur(s)

Association pour l'avancement des sciences et des techniques de la documentation (ASTED)

ISSN

0315-2340 (imprimé)

2291-8949 (numérique)

Découvrir la revue

Citer cet article

Laliberté, J. (1980). Utilisation de la bibliothèque, de la documentation écrite et audiovisuelle au niveau collégial et relations entre le personnel préposé aux ressources et les usagers. Documentation et bibliothèques, 26(2), 99-109. https://doi.org/10.7202/1054249ar

Tous droits réservés (C) Association pour l'avancement des sciences et des techniques de la documentation (ASTED), 1980
Ce document est protégé par la loi sur le droit d'auteur. L'utilisation des services d’Érudit (y compris la reproduction) est assujettie à sa politique d'utilisation que vous pouvez consulter en ligne.

https://apropos.erudit.org/fr/usagers/politique-dutilisation/ 


\section{chronique \\ delarecherche}

\section{Utilisation de la bibliothèque, de la documentation écrite et audiovisuelle au niveau collégial et relations entre le personnel préposé aux ressources et les usagers}

À l'automne de 1976, la Commission des directeurs des services pédagogiques de la Fédération des cégeps confiait à un comité pluridisciplinaire le mandat de réaliser une recherche ayant pour thème général: pratiques pédagogiques et utilisation des ressources dites de soutien à la pédagogie dans les collèges du Québec.

Le 28 février 1980, le comité ${ }^{1}$ rendait public le résultat de ses travaux dans un rapport ${ }^{2}$ qui devrait intéresser non seulement les étudiants et les éducateurs, mais également les personnes qui assument des fonctions et responsabilités dans des bibliothèques, services audiovisuels ou centres de média.

On y trouve, en effet, une foule de données qui permettent de décrire plusieurs aspects significatifs du vécu pédagogique d'un ensemble important de collèges québécois. On y présente et commente les résultats d'une enquête, menée au printemps de 1978, dans dix-huit collèges du Québec: douze cégeps et six collèges privés.

Les réponses fournies par 4374 étudiants, 395 professeurs et 133 préposés

2. Le rapport final prend une double forme: une version intégrale en deux tomes et comptant plus de 700 pages; une version abrégée où se trouvent synthétisés, dans un texte de 80 pages, les résultats jugés les plus significatifs par le comité de coordination de la recherche. Les deux versions portent le même titre: Ressources et pratiques pédagogiques. Le tirage de la version intégrale est limité: la version abrégée a été plus largement diffusée, dans le réseau des collèges tout spécialement. 
aux ressources ${ }^{3}$ renseignent sur l'utilisation d'une variété de ressources didactiques (bibliothèque, documentation écrite ou audiovisuelle, informatique, imprimeriephotocopie) dans des activités d'enseignement et d'apprentissage.

Elles permettent également de caractériser quelques-uns des rapports qui se nouent entre les usagers (étudiants et professeurs) et le personnel chargé d'organiser et d'animer différents secteurs de ressources dites de "soutien à la pédagogie".

Elles jettent enfin de la lumière sur nombre de réalités connexes: les préférences des étudiants concernant les méthodes pédagogiques et la place que celles-ci occupent dans l'enseignement de leurs professeurs; les lectures des étudiants, leurs centres d'intérêt lorsqu'ils lisent ou écoutent des émissions de radio ou de télévision; le lien qu'il peut y avoir entre cette fréquentation des média et leurs activités de formation; les besoins et attentes qu'expriment les étudiants au plan de leur vie intellectuelle et scolaire; les activités de perfectionnement et d'aide pédagogique aux professeurs.

Le rapport final expose de façon détaillée ce qui se dégage des points de vue exprimés par les trois catégories de répondants mis à contribution dans cêtte enquête. Le comité de coordination de la recherche propose aussi au lecteur une série de commentaires qui visent à donner du relief aux données statistiques et à les placer dans un éclairage facilitant leur interprétation. Les auteurs formulent en outre des suggestions à l'intention des personnes ou des groupes désireux de contribuer à une meilleure utilisation des ressources touchées par cette recherche et à un développement de la pédagogie dans les collèges du Québec.

Dans le présent texte, nous voudrions attirer l'attention des lecteurs de

3. Les principales caractéristiques des répondants sont présentées dans un encadré ci-contre. Les lecteurs désireux d'avoir plus d'information sur la méthodologie de l'enquête et sur les modalités de sa réalisation peuvent se référer aux 3 premiers chapitres de la version intégrale, p. 1-114.
Principales caractéristiques des répondants

Dans le groupe des étudiants qui ont rempli le questionnaire, 3335 ou $76,8 \%$ venaient des cégeps et 1010 (ou 23,2\%) des collèges privés; on comptait 2278 femmes $(52,1 \%)$ et 2093 hommes $(47,9 \%)$ avec un âge médian de 19,2 ans; étudiants à temps complet dans une proportion de $93 \%$ suivant leurs cours au collège le jour seulement $(89,8 \%)$ et relevant cle l'enseignement régulier $(92 \%)$, ils se partagaient comme suit: 2415 inscrits à des cours d'enseignement général $(55,2 \%)$ et 1870 à des cours d'enseignement professionnel $(42,8 \%)$.

Du côté des professeurs, 277 ou $70 \%$ enseignaient dans des cégeps et $118(30 \%)$ dans des collèges privés; $135(34,2 \%)$ étaient des femmes et $258(65,3 \%)$ des hommes; l'âge médian était de 33,6 ans tandis que la médiane pour ce qui est des années d'expérience se situait à 8,7 années; à plein temps dans une proportion de $82,5 \%$, les répondants se partagaient par ailleurs comme suit: 248 enseignaient des disciplines comme le français, la philosophie, les sciences et les mathématiques, 158 enseignant une ou plusieurs des techniques biologiques, physiques, humaines, administratives, etc.

Pour ce qui est des préposés, 95 (72\%) venaient des cégeps et $37(28 \%)$ des collèges privés; $61(45,9 \%)$ occupaient une fonction de techniciens, $23(17,3 \%)$ étaient des professionnels, $24(18 \%)$ professionnels responsables d'un service et $16(12 \%)$ cadres; la majorité travaillait à la bibliothèque, à l'audiovidéothèque ou médiathèque, dans le service audiovisuel ou dans un centre intégré de média ou de ressources; l'âge médian des répondants était de 32 ans, la médiane pour les années d'expérience dans la fonction se situant à 4,7 années.

\section{Documentation et bibliothèques}

sur certains résultats de cette enquête qui devraient les rejoindre dans leurs préoccupations professionnelles.

\section{Les étudiants et la documentation écrite}

Les résultats de l'enquête confirment sans équivoque possible la valeur primordiale de la documentation écrite comme ressource au service des activités d'enseignement et d'apprentissage. Parmi les soutiens documentaires, les recueils de textes polycopiés et les manuels d'enseignement occupent une place de choix, comme en témoignent les faits suivants: 
Tableau 1: Lecture: écrits utilisés ou consultés par les étudiants en fonction de leurs études

\begin{tabular}{|c|c|c|c|c|c|c|}
\hline Fréquence & Jamais & Rarement & Souvent & Très souvent & Uniquement & Sans réponse \\
\hline Sorte d'écrits & $(\%)$ & $(\%)$ & $(\%)$ & (\%) & $(\%)$ & $(\%)$ \\
\hline $\begin{array}{l}\text { Livres entiers } \\
\text { (excluant les manuels) }\end{array}$ & $\begin{array}{c}637 \\
(14,6) \\
\end{array}$ & $\begin{array}{r}1512 \\
(34,6) \\
\end{array}$ & $\begin{array}{c}1188 \\
(27,2) \\
\end{array}$ & $\begin{array}{c}741 \\
(16,9) \\
\end{array}$ & $\begin{array}{c}78 \\
(1,8) \\
\end{array}$ & $\begin{array}{c}218 \\
(5,0) \\
\end{array}$ \\
\hline Extraits de livres & $\begin{array}{c}294 \\
(6,7) \\
\end{array}$ & $\begin{array}{c}986 \\
(22,5) \\
\end{array}$ & $\begin{array}{r}1675 \\
(38,3) \\
\end{array}$ & $\begin{array}{r}1223 \\
(28,0) \\
\end{array}$ & $\begin{array}{c}32 \\
(0,7) \\
\end{array}$ & $\begin{array}{r}164 \\
(3,7) \\
\end{array}$ \\
\hline $\begin{array}{l}\text { Articles de revues, } \\
\text { de magazines, de journaux } \\
\text { (imprimés ou microfilmés) }\end{array}$ & $\begin{array}{c}724 \\
(16,6)\end{array}$ & $\begin{array}{r}1525 \\
(34,9) \\
\end{array}$ & $\begin{array}{r}1141 \\
(26,1) \\
\end{array}$ & $\begin{array}{c}838 \\
(19,2) \\
\end{array}$ & $\begin{array}{c}28 \\
(0,6) \\
\end{array}$ & $\begin{array}{r}118 \\
(2,6) \\
\end{array}$ \\
\hline $\begin{array}{l}\text { Encyclopédies, } \\
\text { ouvrages de référence }\end{array}$ & $\begin{array}{c}623 \\
(14,2)\end{array}$ & $\begin{array}{c}1574 \\
(36,0)\end{array}$ & $\begin{array}{c}1225 \\
(28,0)\end{array}$ & $\begin{array}{c}797 \\
(18,2)\end{array}$ & $\begin{array}{c}28 \\
(0,6)\end{array}$ & $\begin{array}{r}127 \\
(2.9) \\
\end{array}$ \\
\hline
\end{tabular}

- Invités à se prononcer sur l'importance qu'ils accordent à une dizaine de moyens d'apprendre allant de la documentation écrite et audiovisuelle aux expériences de travail qui sont en rapport avec les études en passant par les stages, les ateliers ou laboratoires d'enseignement, les musées et les grands organes de communication, les étudiants ont placé au $2 e$ rang les notes de cours et le manuel d'enseignement au 4 e rang. Ce sont les ressources documentaires qu'ils ont le plus souvent utilisées dans les lectures qu'ils ont été amenés à faire en fonction de leurs études.

- Il semble bien, par ailleurs, que les notes de cours, les manuelsmaison, les recueils de textes polycopiés occupent une place éminente dans tous les programmes. D'une part, c'est à propos des notes de cours et des recueils de textes polycopiés qu'on relève le plus petit nombre de variations dans les réponses des étudiants analysées en fonction des familles de programmes auxquelles ces derniers appartiennent. D'autre part, interrogés sur l'importance qu'ils donnent dans la pratique de leur enseignement à trois moyens d'apprendre appartenant à la catégorie des documents écrits, les professeurs ont classé ces moyens dans l'ordre suivant: $1^{\circ}$ notes de cours ou manuels-maison; $2^{\circ}$ documents de la bibliothèque; $3^{\circ}$ manuels édités à l'extérieur du collège.
- On a aussi demandé aux professeurs d'indiquer s'il est nécessaire à leurs étudiants, "pour effectuer leurs travaux et pour réussir", de faire des lectures autres que celles d'un manuel ou de notes de cours. À cela, 96 professeurs $(24,3 \%)$ ont répondu que ce n'est pas nécessaire, $91(23 \%)$ qu'il est impossible de généraliser. Quant à ceux qui ont fourni des précisions, le calcul de la médiane donne 1,58 heures par semaine.

- Dans les lectures qu'ils font en fonction de leurs études, les étudiants disent avoir recours à d'autres sortes d'écrits dans les proportions indiquées dans le Tableau 1.

\footnotetext{
- Des croisements de variables ont permis de constater que les étudiants en sciences pures, sciences de la santé, techniques administratives, techniques biologiques et techniques physiques utilisent plus fréquemment et en beaucoup plus grand nombre des chapitres de manuels ou des manuels entiers que les étudiants en techniques humaines, lettres, sciences humaines et sciences administratives; la situation est, à quelques nuances près, totalement inversée pour ce qui a trait aux livres, extraits de livres et articles de revues ${ }^{4}$
} 
Signalons au passage qu'une analyse plus fouillée des résultats a mis en lumière plusieurs différences de comportements, d'attitudes ou d'opinions suivant les familles de programmes à l'intérieur desquelles se distribuaient les 4374 étudiants qui ont rempli le questionnaire.

À leur arrivée au collège, un grand nombre d'étudiants ne sont pas bien préparés à utiliser les ressources de la documentation écrite.

En majorité (53,7\% ajusté), les étudiants qui ont répondu au questionnaire de l'enquête estiment qu'à leur arrivée au collège, ils savaient déjà assez bien tirer parti de la documentation écrite. Selon les préposés aux ressources cependant, seulement une minorité d'étudiants savent comment exploiter la documentation écrite à leur arrivée au collège. Quant aux professeurs, un très petit nombre exprime son accord avec un énoncé stipulant que "la plupart" des étudiants qui arrivent au collège savent déjà assez bien utiliser "les ressources didactiques" et non pas spécifiquement la documentation écrite. Comment expliquer cette discordance entre les perceptions et les jugements plutôt négatifs des professeurs et des préposés comparativement au point de vue plus positif exprimé par les étudiants? Une analyse plus approfondie des résultats est utile à cet égard:

- si $53 \%$ des étudiants estiment plutôt vraie l'affirmation: "À mon arrivée au collège, je savais déjà assez bien comment tirer profit de la documentation écrite", on en compte $46 \%$ qui trouvent cette affirmation plutôt fausse - ce qui est énorme;

- de plus, seulement $14 \%$ des étudiants ont jugé que l'énoncé était "tout à fait vrai", donc décrivait parfaitement bien la qualité de leur préparation à leur entrée au collège: ainsi, une infime minorité des répondants s'est montrée très catégorique.

Le contraste signalé plus haut est donc plus apparent que réel. Une conclusion s'impose avec netteté: quand ils accèdent au niveau collégial, la plupart des étudiants n'ont pas tout ce qu'il faut pour exploiter au maximum les possibilités que renferme la documentation écrite.

D'ailleurs les étudiants expriment des besoins touchant l'utilisation de la documentation écrite. D'une part, quelle que soit la famille de programmes à laquelle ils appartiennent, deux étudiants sur trois sont d'avis que pour profiter de la documentation écrite et audiovisuelle (l'énoncé ne faisait pas de distinction entre les deux) ils auraient besoin d'une initiation spécifique. Le besoin paraît plus vivement ressenti chez les étudiants des plus gros collèges, ceux qui fréquentent les établissements plus petits étant plus nombreux à soutenir que les indications fournies par les professeurs, à l'occasion des différents cours, leur suffisent amplement. D'autre part, en réponse à une autre question, 49,6\% (ajusté) des étudiants ont affirmé avoir grandement besoin d'aide pour apprendre les principes et les techniques de la lecture rapide, $35,3 \%$ pour dégager ce qui est le plus important dans un texte, $23,3 \%$ pour prendre des notes, $22,9 \%$ pour se servir des ressources qu'offre une bibliothèque et $13,2 \%$ pour travailler avec des fiches.

Les auteurs du rapport estiment donc qu'il y a matière à des interventions diversifiées visant à combler les besoins des étudiants à cet égard. Selon eux, il ne faut pas se laisser trop exclusivement conditionner par l'attirance qu'éprouvent les étudiants pour un apprentissage des principes et techniques de la lecture rapide. Avant toute chose, écrivent-ils, c'est le goût pour la lecture qu'il faut développer, c'est le plaisir de lire qu'il faut susciter. Sur cette base, il devient ensuite possible de fonder une lecture vraiment profitable. Souscrivant à une orientation de base suggérée par la Commission des directeurs de bibliothèques de la Fédération des cégeps, ils proposent de centrer résolument les initiations au travail en bibliothèque sur l'étudiant lui-même, tout en ayant comme objectif de lui apprendre comment se servir efficacement d'une bibliothèque dans une situation de recherche. De cette façon, 
l'information sur les ressources qu'offre la bibliothèque et la façon d'en tirer parti s'inscrivent tout naturellement dans le cadre d'une démarche à travers laquelle l'étudiant acquiert les rudiments d'une bonne méthodologie du travail intellectuel et accède à une plus grande maîtrise des techniques propres à la recherche documentaire.

\section{Les étudiants et la docu. mentation audiovisuelle}

L'audiovisuel exerce un grand attrait sur les étudiants comme en font foi plusieurs résultats de l'enquête.

L'exposé multi-média vient au troisième rang dans la liste des méthodes et formules pédagogiques que les étudiants préfèrent; les grands organes de communication sont, pour une majorité des répondants, "une occasion d'acquérir des connaissances que leurs études ne leur donnent pas" "; très peu d'étudiants 5 s'adonnent à des productions audiovisuelles mais plus de la moitié d'entre eux expriment un grand besoin d'aide pour apprendre comment préparer et produire un document audiovisuel.

Même si une bonne proportion d'étudiants disent accorder de l'importance à la documentation audiovisuelle comme moyen d'apprendre, très rares sont ceux qui ont le réflexe d'v recourir dans leurs études ou leurs travaux.

Près de $89 \%$ (ajusté) des étudiants qui ont collaboré à l'enquête affirment ne s'informer que rarement, voire jamais, des documents audiovisuels auxquels ils pourraient se référer dans leurs études ou travaux scolaires. Pourtant, quand on les

5. Près de $86 \%$ des étudiants qui ont répondu au questionnaire disent ne jamais s'adresser au secteur de l'audiovisuel du collège pour produire eux-mêmes des documents sonores, visuels ou audiovisuels. On trouvera dans la version intégrale du rapport des précisions données par les étudiants qui ont déjà produit des documents audiovisuels: nombre d'heures consacrées à cette tâche, lien entre ces productions et les études, nature des productions (création de documents nouveaux, agencement ou reproduction pure et simple de documents déjà existants), avantages résultant d'une telle expérience. interroge sur l'importance (théorique?) qu'ils accordent à de tels moyens d'apprendre, les résultats prennent une allure sensiblement différente, puisque les réponses se partagent comme suit:

\section{Tableau 2: Importance accordée à la documentation audiovisuelle du collège par les étudiants}

\begin{tabular}{|c|c|}
\hline Aucune, très peu, pent & $\begin{array}{c}\text { Pas mal, grande et } \\
\text { très grande }\end{array}$ \\
\hline $\begin{array}{l}1761 \text { étudiants } \\
(41,7)\end{array}$ & $\begin{array}{l}2461 \text { étudiants } \\
(58,3)\end{array}$ \\
\hline
\end{tabular}

En fait, 28,7\% (ajusté) des répondants disent accorder une grande, voire une très grande importance à cette documentation audiovisuelle comme moyen d'apprendre.

Notons que les professeurs incitent très peu souvent leurs étudiants à s'adresser au centre de documentation audiovisuelle du collège. En effet:

199 professeurs $(50,4 \%)$ ont répondu qu'ils ne le faisaient jamais

74 professeurs $(18,7 \%)$ rarement

74 professeurs $(18,7 \%)$ parfois

28 professeurs $(7,1 \%$; souvent

10 professeurs ( $2,5 \%$ ) très souvent

Les professeurs enseignant dans les collèges les plus populeux ${ }^{6}$ de l'échantillon interviennent plus fréquemment que les autres en ce sens. On a pu constater également que ce sont les professeurs qui ont consacré une plus forte proportion du temps de leurs cours à I'utilisation de l'audiovisuel qui ont le plus favorisé la fréquentation du centre de documentation audiovisuelle par leurs étudiants.

Les étudiants n'en font pas mystère: ils auraient besoin qu'on leur montre comment tirer profit des ressources de la documentation audiovisuelle.

À leur arrivée au collège, les étudiants, dans une proportion de $76,5 \%$, se

6. Population étudiante supérieure à 1000 étudiants. 
sentent très peu préparés à tirer profit de la documentation audiovisuelle et près des deux tiers considèrent qu'ils auraient besoin d'une initiation en bonne et due forme; les professeurs et les préposés aux ressources qui ont rempli les questionnaires de l'enquête se disent d'accord avec cet énoncé.

Chez les professeurs par exemple, $80 \%$ (ajusté) des répondants expriment leur désaccord avec un énoncé stipulant que "la plupart des étudiants qui arrivent au collège savent déjà utiliser assez bien les ressources didactiques" et 75,2\% d'entre eux pensent qu'une véritable initiation leur serait nécessaire. Des croisements de variables ont fait ressortir que les professeurs plus âgés, plus expérimentés et enseignant dans les plus petits collèges ont une opinion plus positive que leurs collègues sur la préparation des étudiants à utiliser les ressources didactiques.

Devant l'engouement dont font preuve les étudiants à l'endroit de l'audiovisuel, les auteurs du rapport se posent plusieurs questions. Ils se demandent s'il faut rapprocher cet engouement de l'intérêt manifesté par les étudiants pour la lecture rapide et le recours à l'ordinateur. Seraientils littéralement fascinés par des techniques et des outils qui sont "dans l'air du temps" et ont pu faire l'objet d'un intense et séduisant marketing? Dans quelle mesure les besoins exprimés sont-ils vraiment ressentis et intériorisés? N'y a-t-il pas danger qu'ils aient été créés artificiellement dans l'esprit des répondants parce que le questionnaire les invitait explicitement à formuler des attentes en ce sens? Faut-il voir dans cet attrait pour l'audiovisuel un exemple de cette propension pour le concret qui caractérise plusieurs des réponses fournies par les étudiants dans cette enquête? Cherche-t-on à se doter d'une panoplie d'instruments à l'aide desquels on pourrait se développer, s'affirmer, s'exprimer de diverses manières? La familiarité avec les grands média de communication est-elle un facteur explicatif? Les auteurs se disent incapables de trancher mais sont d'avis que le phénomène mérite d'être approfondi. Une chose est indéniable: de façon très manifeste, les étudiants posent au personnel des collèges le problème de la pertinence de véritables initiations à l'audiovisuel.

\section{Les étudiants face à la bibliothèque du collège et à son personnel}

Les étudiants fréquentent beaucoup la bibliothèque du collège. Ils s'y rendent surtout pour étudier et travailler ou pour consulter sur place la documentation nécessaire à leurs travaux. En règle générale, ils n'ont pas de difficulté à trouver cette documentation.

\section{- À la question: "A quelle fréquence vous présentez-vous à la bibliothèque du collège?'"}

$\begin{array}{lll}955 \text { étudiants } & (21,8 \%) \text { répondent " "tous les jours" } \\ 1081 & (24,7 \%) & \text { "3 ou } 4 \text { fois par semaine", } \\ 1038 & (23,7 \%) & \text { "1 ou } 2 \text { fois par semaine" } \\ 512 & (11,7 \%) & \text { "1 ou } 2 \text { fois par mois"" } \\ 179 & (4,1 \%) & \text { "1 ou } 2 \text { fois par année" } \\ 121 & (2,8 \%) & \text { "jamais" } \\ 408 & (9,3 \%) & \text { "je ne saurais dire" }\end{array}$

Les étudiants du secteur général s'y rendent beaucoup plus fréquemment que ceux du professionnel comme l'illustrent ces statistiques:

\section{Tableau 3: Se rendent à la bibliothèque du collège au moins 3 ou 4 fois par semaine}

\begin{tabular}{|lrc|}
\hline Secteur & & \\
\hline & $N$ & $\%$ \\
Général & 1378 & 57,5 \\
Professionnel & 615 & 35,1 \\
Total & 1993 & 48,0 \\
\hline
\end{tabular}

Invités à se prononcer sur une série de sept motifs possibles de fréquenter la bibliothèque de leur collège, les étudiants ont établi la hiérarchie que voici: 


\section{Tableau 4}

\begin{tabular}{|c|c|c|c|}
\hline & Motif & $\begin{array}{l}\text { Nombre d'étudiants qui } \\
\text { se rendent souvent ou } \\
\text { très souvent à la biblio- } \\
\text { theque pour ce motif }\end{array}$ & $\%$ ajusté \\
\hline 1. & $\begin{array}{l}\text { La biblicthèque est pour moi un bon endroit } \\
\text { pour étudier ou pour réaliser mes travaux } \\
\text { (salle d'étude). }\end{array}$ & 2810 & 66,1 \\
\hline 2. & $\begin{array}{l}\text { Je viens consulter sur place la documentation } \\
\text { nécessaire à mes travaux. }\end{array}$ & 2685 & 63,1 \\
\hline 3. & $\begin{array}{l}\text { La biblioth ièque nous fournit les locaux dont } \\
\text { nous avons besoin pour des travaux d'équipe. }\end{array}$ & 1724 & 42,1 \\
\hline 4. & $\begin{array}{l}\text { Je viens seulement emprunter la documen- } \\
\text { tation dont j'ai besoin pour mes études ou } \\
\text { pour mes loisirs. }\end{array}$ & 1583 & 37,7 \\
\hline 5. & $\begin{array}{l}\text { Je viens prendre connaissance des nou- } \\
\text { veautés, feuilleter livres, journaux et revues. }\end{array}$ & 901 & 21,3 \\
\hline 6. & $\begin{array}{l}\text { Entre deux cours, je me rends à la } \\
\text { bibliothèque pour occuper le temps ou } \\
\text { pour me détendre. }\end{array}$ & 597 & 14,3 \\
\hline 7. & $\begin{array}{l}\text { Je réponds à une convocation ou à une } \\
\text { invitation d'un de mes professeurs qui donne } \\
\text { son cours ou organise des activités pédago- } \\
\text { giques dans l'un ou l'autre des locaux } \\
\text { de la bibliothèque. }\end{array}$ & 245 & 6,0 \\
\hline
\end{tabular}

- Dans une proportion de 66,3\% (ajusté), les étudiants disent n'avoir que rarement sinon pas du tout de difficulté à trouver au collège la documentation nécessaire à un travail proposé par l'un ou l'autre de leurs professeurs. Cependant les étudiants fréquentant les collèges les plus petits ${ }^{7}$ de l'échantillon éprouveraient plus de difficulté que ceux des collèges plus populeux.

Dans leur études ou leurs travaux scolaires, les étudiants font très peu souvent appel au personnel de la bibliothèque.
- Quand ils ont recours à la bibliothèque ou au centre de ressources didactiques du collège pour étudier ou approfondir une question, les étudiants d'une façon générale ne sollicitent jamais ou alors rarement les avis, commentaires et suggestions du personnel de la bibliothèque sur le sujet même de leur recherche mais ils sont plus nombreux à demander de l'aide pour trouver la documentation qui leur serait utile comme le montrent bien les données suivantes:

\section{Tableau 5}

\begin{tabular}{|c|c|c|c|c|c|}
\hline \multirow[b]{2}{*}{ - } & Jamais & Rarement & Souvent & $\begin{array}{c}\text { Très } \\
\text { souvent }\end{array}$ & $\begin{array}{c}\text { Sans } \\
\text { réponse }\end{array}$ \\
\hline & $N \quad(\%)$ & $(\%)$ & $\mathrm{N} \quad(\%)$ & $N \quad(\%)$ & $N \quad(\%)$ \\
\hline $\begin{array}{l}\text { Je demande à un membre du personnel } \\
\text { de la bibliothèque de m'aider à trouver } \\
\text { la documentation qui me serait utile }\end{array}$ & $\begin{array}{l}1442 \\
(33,0)\end{array}$ & $\begin{array}{l}2029 \\
(46,4)\end{array}$ & $\begin{array}{c}652 \\
(14,9)\end{array}$ & $\begin{array}{c}131 \\
(3,0)\end{array}$ & $\begin{array}{c}120 \\
(2,7)\end{array}$ \\
\hline $\begin{array}{l}\text { Je discute avec un membre du } \\
\text { personnel du sujet de ma recherche et } \\
\text { je sollicite ses avis, commentaires et } \\
\text { suggestions }\end{array}$ & $\begin{array}{l}2895 \\
(66,2)\end{array}$ & $\begin{array}{l}1100 \\
(25,1)\end{array}$ & $\begin{array}{l}202 \\
(4,6)\end{array}$ & $\begin{array}{c}45 \\
(1,0)\end{array}$ & $\begin{array}{c}132 \\
(3,1)\end{array}$ \\
\hline
\end{tabular}

7. Population étudiante inférieure à 1000 étudiants.

Différence établie à l'aide du Chi carré. 
- De l'avis des préposés aux ressources $(\mathrm{N}=68$ répondants pour $51,1 \%$ et $81,9 \%$ ajusté), c'est la minorité des étudiants qui discutent avec un membre du personnel du sujet de leur recherche et sollicitent ses avis, commentaires et suggestions. Les opinions sont plus partagées sur l'autre question: selon 41 préposés (46,6\% ajusté), c'est une minorité d'étudiants qui demandent de l'aide pour trouver la documentation utile ou pertinente, tandis que pour 47 préposés $(53,4 \%)$ c'est une majorité d'étudiants qui sollicitent pareille aide. Le point de vue des préposés vient donc corroborer les réponses des étudiants.

Dans les commentaires libres des étudiants, les auteurs du rapport ont cru se rendre compte d'une chose: parmi les diverses ressources dites de soutien que le collège met à leur disposition, c'est manifestement de la bibliothèque que le plus grand nombre d'étudiants peuvent parler en connaissance de cause. C'est à son sujet également qu'ils expriment le plus de satisfaction. Comment expliquer qu'ils fassent si peu appel à son personnel dans leurs travaux scolaires? A cela, ils proposent quelques éléments d'interprétation qui, loin de prétendre épuiser la gamme des explications possibles, pourraient peut-être servir d'amorce à une réflexion qui examinerait plus en profondeur cet état de fait.

- À y regarder de près, les statistiques concernant le nombre des étudiants qui demandent de l'aide à un membre du personnel de la bibliothèque pour trouver la documentation pertinente à leur travail n'a pas de quoi étonner. Dans la mesure où l'on sait se débrouiller en bibliothèque, on n'a pas nécessairement à recourir fréquemment aux services de son personnel. Notons que près des deux tiers des répondants font tout de même appel à l'occasion au personnel pour trouver la documentation dont ils ont besoin.

- Qu'on ne discute pas plus souvent avec des membres du personnel de la bibliothèque du sujet de sa recherche, qu'on ne recherche pas les avis, commentaires et suggestions de ces préposés, cela peut tenir à plusieurs facteurs: a) on n'en sent pas le besoin, ayant à sa disposition, si cela s'avère nécessaire, plusieurs autres types d'interlocuteurs à qui s'adresser, notamment les camarades et les professeurs; b) on ne considère pas cette catégorie de personne ${ }^{8}$ comme une ressource qui pourrait être utilisée à cette fin (conception qu'on se fait du rôle et des fonctions des bibliothécaires et des bibliotechniciens); c) on a vécu de mauvaises expériences: dans des commentaires libres, des étudiants critiquent l'attitude de certains préposés qui ne seraient pas tellement accueillants quand on sollicite leur concours.

\section{Les relations entre les professeurs et les préposés aux ressources}

Lorsqu'ils préparent un cours, font l'essai d'une innovation, doivent résoudre des problèmes particuliers ou surmonter des difficultés dans la pratique de leur enseignement, les professeurs s'adressent très peu aux préposés à la documentation écrite ou audiovisuelle.
- Plus des trois quarts ( \% ajusté) des professeurs interrogés disent ne jamais s'adresser au personnel de la bibliothèque ou du secteur audio- visuel dans les circonstances ou pour les fins énumérées plus haut. C'est avec leurs pairs et les respon-

8. Environ $42 \%$ des étudiants estiment qu'ils n'ont pas suffisamment de conversations ou de discussions avec le personnel de la bibliothèque ou du service audiovisuel au sujet de leurs études. Un pourcentage à peu près équivalent n'a pas répondu à une interrogation en ce sens, ce qui pourrait, entre autres hypothèses explicatives, refléter une conviction qu'auraient ces étudiants qu'ils ne peuvent pas recourir aux bons offices de ces personnes. Ou alors faut-il y voir un désintérêt pour une communication accrue avec cette catégorie de personnel? 
sables de la coordination départementale qu'ils entrent alors surtout en communication ${ }^{9}$.

- Remarquons cependant qu'entre 37 et $41 \%$ des mêmes professeurs jugent insuffisants les échanges qu'ils ont avec ces deux catégories de préposés. Les répondants en provenance des collèges les plus populeux sont proportionnellement plus nombreux à exprimer ce point de vue. C'est aussi dans ces collèges qu'on compte le plus grand nombre de professeurs qui expliquent l'insuffisance de leurs contacts avec les préposés aux ressources par une carence d'information sur le genre de services que ces personnes pourraient rendre. Cette convergence de faits donne à penser que dans les collèges plus populeux, on aurait particulièrement intérêt à étudier de plus près l'état et le mode de fonctionnement des relations entre le corps professoral et le personnel des ressources.

Le réseau de communication entre préposés aux ressources et professeurs revêt le plus souvent un caractère informel.

D'une part, quand on analyse les précisions fournies par les préposés qui ont eu l'occasion d'apporter une aide aux professeurs, on constate que l'initiative est généralement venue du ou des professeurs; la collaboration avec un seul professeur a été la modalité la plus fréquemment pratiquée, la collaboration avec l'ensemble du corps professoral ou quelques départements étant les modalités les moins souvent mentionnées.

D'autre part, c'est surtout à travers des conversations ou démarches sans caractère officiel que les professeurs sont intervenus pour exprimer leurs opinions sur l'orientation et l'organisation des ressources didactiques mises à leur disposition par le collège. De même, lorsqu'on a interrogé les préposés sur les initiatives ou mesures prises pour identifier et clarifier

9. Version intégrale, tome 2, p. 392-402. les besoins des usagers (individus ou groupes), les conversations ou démarches sans caractère officiel sont également venues en tête de liste avec 186 mentions sur 539 , soit $34,5 \%$ de toutes les mentions

Ainsi les interactions fondées sur des mécanismes institutionnels bien identifiés comme tels (qu'il s'agisse d'enquêtes, de sondages ou de comités) n'apparaissent pas tellement répandues ou fréquentes, du moins dans les collèges où s'est effectuée cette recherche.

Plusieurs réponses tant des professeurs que des préposés aux ressources témoignent de dispositions d'esprit propres à favoriser une plus grande interaction entre les uns et les autres.

Ainsi professeurs et préposés s'entendent pour affirmer que le personnel affecté à la documentation écrite et audiovisuelle ne devrait pas se borner à remplir des commandes de matériel ou à donner seulement des renseignements ou conseils relevant de sa seule compétence technique. Nombre de professeurs semblent souhaiter que cette catégorie de préposés fasse preuve de plus d'initiative en offrant ses services à tous les professeurs, et non pas seulement à ceux qui s'adressent à eux; cette attente s'exprime avec plus de netteté encore dans les gros collèges de l'échantillon.

Par ailleurs, les professeurs désirent jouer un rôle actif dans les processus qui visent à donner une orientation précise aux ressources didactiques ou à déterminer les modalités de leur organisation, en particulier pour ce qui a trait aux objets suivants:

$1^{\circ}$ le choix ou la sélection de la documentation écrite;

$2^{\circ}$ les besoins des usagers;

$3^{\circ}$ le choix ou la sélection des documents et de l'équipement audiovisuels.

Ils ont aussi signifié de façon très nette leur intérêt et leur motivation à collaborer avec le personnel des ressources. En effet, $64 \%$ (ajusté) des professeurs se disent passablement ou très motivés à collaborer à l'amélioration des ressources didactiques 
avec les personnes qui en ont actuellement la charge et $56 \%$ (ajusté) estiment que les professeurs qu'ils connaissent manifestent passablement ou beaucoup d'intérêt pour l'amélioration des ressources didactiques du collège.

Professeurs et préposés identifient un certain nombre de facteurs qui empêchent ou compromettent une plus grande communication entre les uns et les autres.

On a interrogé les deux catégories de répondants sur les raisons qui pourraient expliquer une communication insuffisante touchant l'orientation et l'organisation des ressources didactiques du collège. 146 professeurs (37\%) et 64 préposés (48\%) estiment qu'il y a suffisamment d'échanges et de collaboration à ce sujet. Les autres s'entendent pour pointer trois principaux obstacles à surmonter si l'on veut instaurer une interaction plus dynamique:

$1^{\circ}$ les habitudes acquises;

$2^{\circ}$ les structures et les méthodes de communication utilisées;

$3^{\circ}$ le peu d'intérêt des professeurs à l'égard des comités purement consultatifs.

En somme, des facteurs qui mettent en cause mentalités et façons de faire, qui touchent aux modes de fonctionnement et d'organisation, qui rejoignent la problématique plus vaste de la participation et du partage du pouvoir dans le respect des compétences respectives.

On a aussi cherché à savoir ce qui pouvait empêcher les professeurs de communiquer avec certaines personnesressources dont ils souhaiteraient recevoir de l'aide, soit dans la préparation de leurs cours, soit à l'occasion de difficultés qu'ils éprouvent. 158 professeurs (40\%) ont estimé que la question ne les concernait pas et 30 préposés $(22,6 \%)$ considèrent qu'il y a suffisamment de communication à cet égard. Quant aux raisons mises de l'avant par ceux qui déplorent une insuffisance de relations, on constate le phénomène suivant: si les professeurs et les préposés s'entendent pour souligner des lacunes au plan de l'information, les premiers ont tendance à reprocher au personnel des ressources un manque d'intérêt et de disponibilité, tandis que les seconds accordent beaucoup d'importance au fait que, selon eux, les professeurs ne veulent pas que les préposés interviennent en matière de pédagogie.

\section{Conclusion}

Les données que nous venons de mettre en relief ne sont pas les seules qui soient de nature à intéresser des professionnels de la documentation. Elles suffisent, nous semble-t-il, à faire ressortir I'utilité d'une analyse plus approfondie des résultats de la recherche sur les Ressources et pratiques pédagogiques au niveau collégial. L'enquête réalisée dans dix-huit collèges du Québec contribue sans doute à une meilleure connaissance de la réalité mais elle peut aussi - et les auteurs du rapport insistent beaucoup là-dessus devenir une incitation à l'action. Le comité de coordination propose plusieurs pistes en ce sens, notamment les trois suivantes:

1. Dans plusieurs collèges, on gagnerait à se donner de véritables politiques de sensibilisation et d'initiation aux ressources ou à réexaminer soigneusement celles qui sont en vigueur.

Le manque d'information sur les ressources et la revendication d'une initiation aux ressources reviennent très fréquemment dans la série des commentaires libres recueillis au cours de l'enquête. Le dépouillement des questionnaires a révélé que nombre d'étudiants étaient ignorants des services disponibles au collège.

D'autre part, dans quelques collèges tout au moins, il ne serait pas superfiu de bien délimiter les politiques d'accessibilité des étudiants aux ressources et services qu'offre le secteur audiovisuel. Certains témoignages d'étudiants donnent en effet à penser qu'en certains milieux il subsiste beaucoup d'ambiguiité et de flottement à ce sujet. II s'impose- 
rait donc que les politiques adoptées soient clairement énoncées et portées à la connaissance de tous les intéressés.

Enfin, selon les auteurs du rapport, dans tout ce travail d'information, de sensibilisation et d'initiation aux ressources, le souci des adultes avec leur situation particulière et leurs besoins propres devrait retenir l'attention et mobiliser des énergies toute spéciales.

2. L'information, la sensibilisation et l'initiation aux ressources doivent dépasser les perspectives étroites du maniement des appareils, du recours à des techniques, etc. pour aborder de front des questions fondamentales tels le caractère instrumental des ressources et le potentiel énorme qu'elles recèlent au plan pédagogique.

Les auteurs du rapport font remarquer qu'en matière d'utilisation des ressources technologiques, on oscille entre deux tendances extrêmes: a) "mythifier" ces ressources, attendre d'elles plus qu'elles ne peuvent donner, céder à la mode du gadget ou de la quincaillerie apparemment la plus révolutionnaire et la plus prometteuse; b) se méfier d'elles, les rejeter sans examen, par crainte, par inertie ou par traditionalisme et convervatisme. Ils plaident en faveur d'une meilleure utilisation de ces ressources, une utilisation trouvant place, fonction, utilité à l'intérieur d'une véritable stratégie d'enseignement ou d'apprentissage et renvoient à quelques ouvrages qui illustrent bien cette préoccupation.

3. Les collèges gagneraient à se donner les politiques, les structures et les mécanismes susceptibles d'assurer une plus grande concertation de toutes les personnes mises en cause dans l'oeuvre d'éducation et dans les activités pédagogiques.
Sans une action concertée des pédagogues et du personnel préposé aux ressources, comment prétendre que l'on tire tout le parti possible des compétences nombreuses et complémentaires dont dispose un collège? Les auteurs du rapport estiment que les relations entre usagers et préposés pourraient être plus nombreuses et de plus grande signification.

Ayant constaté que l'enquête même a permis d'identifier des dispositions d'esprit favorables à une plus grande interaction, ils invitent les lecteurs et utilisateurs du rapport à étudier ces problèmes et défis en recourant à une approche de type systémique, en considérant le collège comme un système, lui-même constitué de plusieurs sous-systèmes concourant chacun à sa façon mais en liaison organique avec les autres à l'atteinte des objectifs de l'institution. Cependant, ils laissent à l'ingéniosité des personnels de collèges le soin de préciser les modèles d'interaction les plus appropriés.

Cette attitude est d'ailleurs typique: tout au long du rapport, on trouve des invitations à pousser plus loin l'analyse et la réflexion, des exhortations à une action dont on indique le sens général sans entrer dans les modalités susceptibles de varier considérablement d'un collège à l'autre, d'un groupe de personnes à l'autre.

Ainsi le rapport final sur la recherche Ressources et pratiques pédagogiques marque le point d'arrivée d'une démarche longue et exigeante mais il pourrait servir de point de départ à quantité d'initiatives qui prolongeraient, donneraient encore plus de poids, de signification, de pertinence aux résultats obtenus.

\section{Jacques Laliberté}

Centre d'animation, de développement et de recherche en éducation (C.A.D.R.E) Montréal 